\title{
Photoelectron spectroscopy of $\mathrm{O}^{-}$at $266 \mathrm{~nm}$ : Ratio of ground- and excited-state atomic oxygen production and channel-resolved photoelectron anisotropy parameters
}

\author{
C. Domesle, ${ }^{1}$ B. Jordon-Thaden, ${ }^{1}$ L. Lammich, ${ }^{2}$ M. Förstel,,${ }^{1,3}$ U. Hergenhahn, ${ }^{3}$ A. Wolf,,${ }^{1}$ and H. B. Pedersen ${ }^{2, *}$ \\ ${ }^{1}$ Max-Planck-Institut für Kernphysik, DE-69117 Heidelberg, Germany \\ ${ }^{2}$ Department of Physics and Astronomy, Aarhus University, DK-8000 Aarhus C, Denmark \\ ${ }^{3}$ Max-Planck-Institut für Plasmaphysik, EURATOM Association, DE-85748 Garching, Germany
}

(Received 1 July 2010; published 3 September 2010)

\begin{abstract}
The photodetachment dynamics of the atomic oxygen anion $\mathrm{O}^{-}$has been investigated at $266 \mathrm{~nm}(4.67 \mathrm{eV})$ by photoelectron detection in a crossed-beam experiment using a magnetic-bottle electron spectrometer. Taking explicit advantage of the Doppler shift imposed by the moving ion beam on the photoelectron energies, we report both the final-state branching ratio and photoelectron angular distributions. After photoabsorption at $266 \mathrm{~nm}$, the formed electron-oxygen scattering state disintegrates, forming either the excited ${ }^{1} D$ or the ground ${ }^{3} P$ state of oxygen with a partition of ${ }^{1} D:{ }^{3} P=0.32 \pm 0.06$. The detachment leading to the production of $\mathrm{O}\left({ }^{3} P\right)$ shows an angular distribution of photoelectrons characterized by $\beta_{P}=0.00 \pm 0.10$ mimicking a pure s-wave detachment, while the detachment into excited $\mathrm{O}\left({ }^{1} D\right)$ occurs with $\beta_{D}=-0.90 \pm 0.10$, giving direct evidence of interference between the outgoing $s$ and $d$ waves.
\end{abstract}

DOI: 10.1103/PhysRevA.82.033402

PACS number(s): $32.80 . G c$

\section{INTRODUCTION}

The photodetachment of the oxygen anion is an important model case for understanding effects of correlated electron motion in an open-shell system and, moreover, plays a role for the oxygen chemistry in the Earth's and other planetary atmospheres [1]. Under these aspects the photodetachment process has been the subject of both experimental [2-14] and theoretical [3,15-25] investigations for more than half a century.

A simplified illustration of the oxygen photodetachment process is displayed in Fig. 1. Through photoabsorption, a continuum-electron-oxygen scattering state is generated, which (within the $L S$-coupling description) has components of ${ }^{2} P,{ }^{2} D$, and ${ }^{2} S$ symmetry. The relative population of these components depends on the initial state of the anion and thereby reflects electron correlation in its ${ }^{2} P$ ground state. The scattering state is characterized by various final states composed of a free electron and a neutral oxygen atom. The partitioning of intensity among the different final states reflects the couplings among the scattering wave components due to relativistic (e.g., spin-orbit) or anisotropic electron-neutral (e.g., polarization) interactions as well as the interference between waves leading to the same final state. The integral effects of the photoabsorption and the scattering reaction are observationally manifest both through the angular distribution of photoelectrons, described by the anisotropy (asymmetry) parameter $\beta$, and through the relative populations $F_{X}(X=$ $\left.{ }^{3} P_{J},{ }^{1} D_{J},{ }^{1} S_{J}\right)$ of final excited states of the neutral $\mathrm{O}$ atom, as identified by the photoelectron energy.

One class of earlier experimental investigations has focused on determining the cross section for the oxygen photodetachment both on an absolute scale $[4,10,14]$ and as a function of photon energy $[4,11,12]$ marked with a shaded area in Fig. 1. In particular the energy region near and above the first detachment

\footnotetext{
*hbjp@phys.au.dk
}

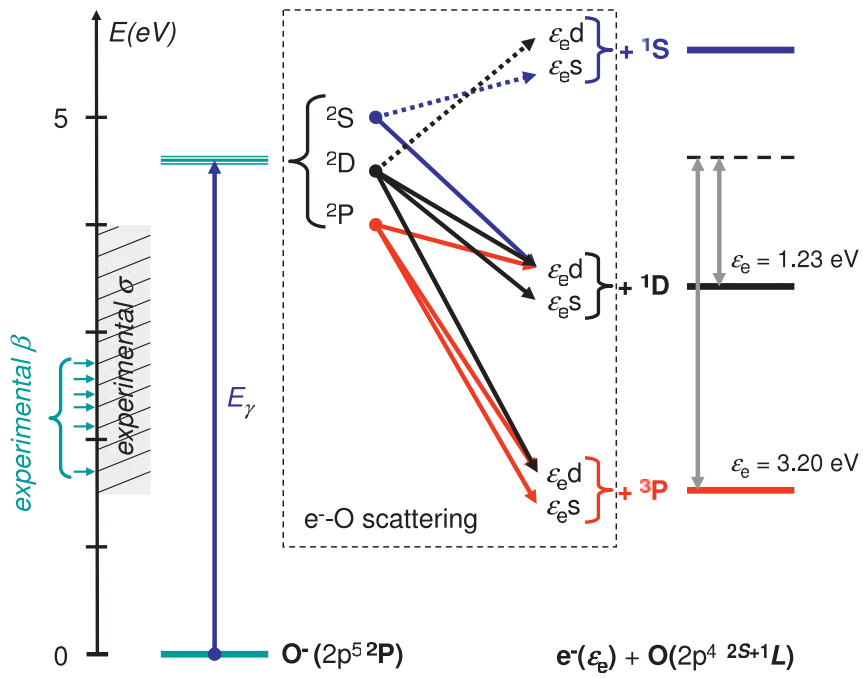

FIG. 1. (Color online) Illustration of the photodetachment of atomic oxygen at $266 \mathrm{~nm}$. The shaded area along the energy axis indicates the region of experimentally known cross section $[4,11,12]$, while the arrows indicate photon energies where electron angular distributions and branching ratios into the ${ }^{3} P_{J}$ multiplet states have previously been measured [5-8].

threshold $\left({ }^{2} P_{J=1 / 2,3 / 2} \rightarrow{ }^{3} P_{J=0,1,2}\right)$ has been addressed to establish an accurate value for the electron affinity $[2,11,13]$, the energies of the individual fine structure transitions [11,12], as well as the precise energy dependence of the photodetachment cross section [4,12]; in total the photodetachment cross section is experimentally determined up to photon energies of $4 \mathrm{eV}$, and only a single experimental study [4] has addressed the photodetachment cross section in the region where production of the ${ }^{1} D$ state of oxygen is energetically allowed. Another class of experiments (green arrows in Fig. 1) has addressed explicitly the dynamics of the electron-oxygen scattering process following the photoabsorption, by measurements of the angular distribution of the emerging photoelectrons [5-8] and the branching ratios for population of the different fine 


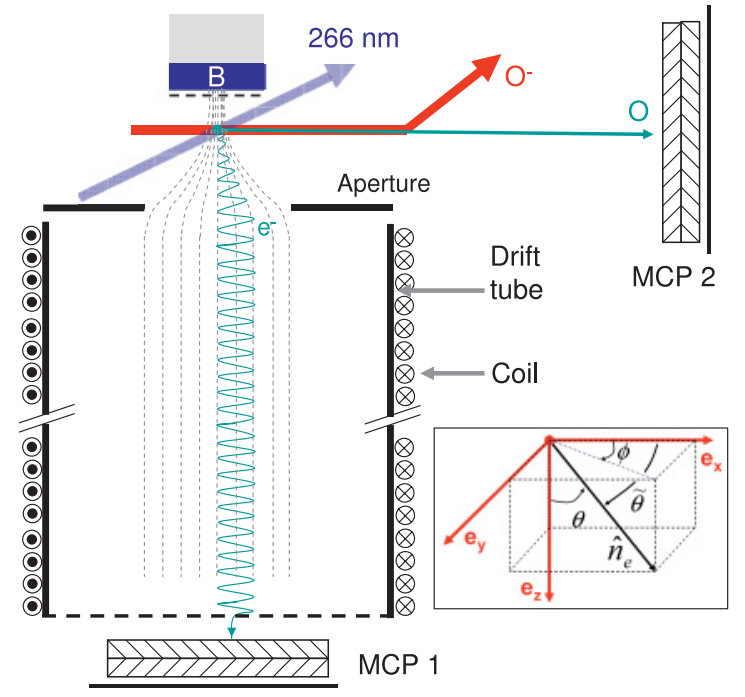

FIG. 2. (Color online) Illustration (not to scale) of the interaction region with the magnetic-bottle photoelectron spectrometer.

structure components of the final oxygen state [6,7,9]. So far, such investigations have focused entirely on the ${ }^{2} P_{J=1 / 2,3 / 2} \rightarrow$ ${ }^{3} P_{J=0,1,2}$ transitions as indicated in Fig. 1 .

In this paper we report on an investigation of the electronoxygen scattering states formed by photodetachment at $266 \mathrm{~nm}$, thus with an internal energy where the production of excited state $\mathrm{O}\left({ }^{1} D\right)$ and ground state $\mathrm{O}\left({ }^{3} P\right)$ compete, and where so far only theoretical calculations have been available [15-23]. The experiment uses a fast beam of ions crossed by a pulsed laser beam inside a magnetic-bottle photoelectron spectrometer (see Refs. [26-28]). Exploiting the Doppler shift imposed on the energies of the emitted electrons by the movement of the ion beam, the experiment maps information on both the final atomic state, ${ }^{1} D$ or ${ }^{3} P$, and the anisotropy of the electron emission into the observable time-of-flight (TOF) distribution of the photoelectrons.

\section{EXPERIMENT}

\section{A. Setup}

The experiment was performed at the ion beam facility TIFF [29] (trapped ion fragmentation with a free-electron laser) installed at the free-electron laser (FLASH) facility [30] at DESY in Hamburg. For the present study we used this ion beam infrastructure, dedicated to crossed-beam investigations of ion-photon interactions, in combination with a Nd:YAG laser system.

At the TIFF setup, a collimated ion beam of $\sim 2 \mathrm{nA}$ of oxygen anions with a kinetic energy of $E_{0}=4.45 \mathrm{keV}\left(v_{i}=\right.$ $232 \mathrm{~mm} / \mu \mathrm{s}$ ) was extracted from a hollow cathode ion source operated with a gas inlet of pure water vapor. From TIFF, ion pulses with a width of $10 \mu$ s were transported through a recently built ion beam line to an interaction zone equipped with a magnetic-bottle photoelectron spectrometer.

A schematic drawing of the ion-photon interaction region and the electron spectrometer is shown in Fig. 2; both the ion and the photon beam move in the horizontal plane, and the central axis of the electron spectrometer is vertical. At the interaction point the ion pulses were crossed at $90^{\circ}$ by a horizontally polarized laser with $266 \mathrm{~nm}$ wavelength, $\sim 5 \mathrm{~ns}$ pulse width and a mean pulse intensity of $\sim 50 \mu \mathrm{J}$, corresponding to $\sim 7 \times 10^{13}$ photons per pulse. For each laser pulse, the relative intensity and the precise arrival time at the interaction point were monitored by a fast photodiode, while the absolute mean pulse intensity was recorded using a slower thermal power meter.

The spatial profiles of the two beams were determined inside the electron spectrometer with a movable beam flag [28]. The ion beam could be represented by Gaussian distributions in both horizontal and vertical directions with widths (rms) of 1.5 and $1.4 \mathrm{~mm}$, respectively; the photon beam was nearly circular with a Gaussian width of $0.9 \mathrm{~mm}$ : thus, a total of $\sim 40$ ions were irradiated in each laser pulse.

The magnetic-bottle spectrometer (see Fig. 2) consists of a small permanent magnet with a solid iron cone located $\sim 1 \mathrm{~cm}$ above the interaction volume for concentrating the magnetic field lines, and a $600 \mathrm{~mm}$ long drift tube equipped with a solenoid magnet starting $\sim 5 \mathrm{~cm}$ below the interaction zone. A $40 \mathrm{~mm}$ multichannel plate detector (MCP 1), positioned at the end of the drift tube, was used for electron detection. In this type of arrangement [26,27,31-33], photoelectrons are emitted in a strong field (here $\sim 50 \mathrm{mT}$ ) and magnetically bent downward into the drift tube where a constant, weak magnetic guiding field $(\sim 2 \mathrm{mT})$ is maintained by the solenoid. The primary $\mathrm{O}^{-}$beam passed a set of electrostatic deflection plates $20 \mathrm{~cm}$ downstream from the interaction region, bending it into a Faraday cup. The TOF of neutral oxygen atoms produced in the photodetachment process or in collisions with the residual gas was registered by another $40 \mathrm{~mm} \mathrm{MCP}$ detector (MCP 2) located $\sim 50 \mathrm{~cm}$ behind the interaction region.

\section{B. Photoelectron TOF distribution}

In the rest frame of the reactants, $\mathrm{O}^{-}+\gamma$, a photodetachment event at $\lambda_{\gamma}=266 \mathrm{~nm}$ will lead to a photoelectron emerging with a kinetic energy of

$$
\varepsilon_{e}=\left(E_{\gamma}-E_{D, \mathrm{O}}-E_{X}\right)\left(\mu / m_{e}\right),
$$

where $E_{\gamma}=4.67 \mathrm{eV}$ is the photon energy, $E_{D, \mathrm{O}}=1.46 \mathrm{eV}$ is the detachment threshold energy of oxygen, $E_{X}$ represents the excitation energy of the residual neutral oxygen, that is, $E_{3} P$ $=0$ and $E_{1_{D}}=1.97 \mathrm{eV}$, and $\mu=m_{\mathrm{O}} m_{e} /\left(m_{\mathrm{O}}+m_{e}\right) \approx m_{e}$. The distribution of emission angles of the photoelectrons can generally be described by the formula [25]

$$
P(\tilde{\theta}, \beta) d(\cos \tilde{\theta})=\left[\frac{1}{2}-\frac{\beta}{4}+\frac{3 \beta}{4} \cos ^{2} \tilde{\theta}\right] d(\cos \tilde{\theta}),
$$

where $\tilde{\theta}$ is the angle of emission relative to the laser polarization (here $\boldsymbol{\epsilon}_{\gamma}=\mathbf{e}_{x}$ ) and $\beta \in[-1 ; 2]$ is the anisotropy parameter.

Under the present experimental conditions, the fast motion of the detaching $\mathrm{O}^{-}$ions modifies the observable electron energies in the laboratory frame by a Doppler shift that depends explicitly on the angle of emission relative to the ion motion. Thus, neglecting the momentum of the photon with respect to that of the ion, i.e., $E_{\gamma} /\left(m_{i} v_{i} c\right) \approx 0$, and using the coordinates indicated in Fig. 2, a photoelectron emitted from an oxygen 
anion moving at speed $v_{\mathrm{i}}$ emerges with a total laboratory velocity $\mathbf{v}_{\mathbf{e}}^{\mathbf{L}}$ given by

$$
\mathbf{v}_{\mathrm{e}}^{\mathbf{L}}=v_{\mathrm{i}} \mathbf{e}_{\mathbf{x}}+v_{\mathrm{e}} \hat{\mathbf{n}}_{e}=\mathbf{v}_{\mathrm{ei}}^{\perp}+\mathbf{v}_{\mathrm{ei}}^{\|},
$$

where $v_{\mathrm{e}}=\sqrt{2 \varepsilon_{\mathrm{e}} / m_{\mathrm{e}}}$ and $\hat{\mathbf{n}}_{e}=\sin \theta \cos \phi \mathbf{e}_{\mathbf{x}}+\sin \theta \sin \phi \mathbf{e}_{\mathbf{y}}+$ $\cos \theta \mathbf{e}_{\mathbf{z}}$ is the unit vector in the direction of electron emission in the rest frame of $\mathrm{O}^{-}$with $\theta$ and $\phi$ representing the inclination and azimuth angles of a spherical coordinate system. The coordinate system is defined with $\mathbf{v}_{\mathrm{ei}}^{\perp}$ in the $x y$ plane and $\mathbf{v}_{\mathrm{ei}}^{\|_{\mathrm{i}}}$ parallel to the $z$ axis.

After detachment, the photoelectron is bent in the magnetic field of the electron spectrometer, thereby effectively converting part of its initial momentum in the direction transverse to the magnetic field, $m \mathbf{v}_{\text {ei }}^{\perp}$ with $v_{\text {ei }}^{\perp}=$ $\sqrt{v_{i}^{2}+v_{e}^{2} \sin ^{2} \theta+2 v_{i} v_{e} \sin \theta \cos \phi}$, into momentum in the direction parallel to the magnetic field, a process known as parallelization [26].

In the simplest idealized representation, we assume all photoelectrons to be instantaneously parallelized to the magnetic field with a complete transfer of transverse to longitudinal momentum, while maintaining their kinetic energy. Under this assumption a photoelectron appears immediately with its final longitudinal velocity of $v_{\mathrm{ef}}^{\|}=\sqrt{\left(v_{\mathrm{ei}}^{\|}\right)^{2}+\left(v_{\mathrm{ei}}^{\perp}\right)^{2}}=$ $\sqrt{v_{i}^{2}+v_{e}^{2}+2 v_{i} v_{e} \sin \theta \cos \phi}$. Moreover, since the laser is horizontally polarized, $\boldsymbol{\epsilon}_{\gamma} \cdot \hat{\mathbf{n}}_{\mathbf{e}}=\sin \theta \cos \phi=\cos \tilde{\theta}$. The time of flight of a photoelectron to MCP 1 can thus be written as

$$
t=L / \sqrt{v_{i}^{2}+v_{e}^{2}+2 v_{i} v_{e} \cos \tilde{\theta}}
$$

where $L$ is the total distance from the interaction point to the detector surface ( $L=653 \mathrm{~mm}$ ). Finally, combining Eqs. (2) and (4), the following formula for the photoelectron TOF distribution can be derived:

$$
\begin{aligned}
P\left(t, v_{e}, v_{i}, \beta\right) d t & =\frac{1}{2}\left\{1-\frac{\beta}{2}+\frac{3 \beta}{8}\left(\frac{v_{e}}{v_{i}}\right)^{2}\left[1+\left(\frac{v_{i}}{v_{e}}\right)^{2}-\left(\frac{L}{t v_{e}}\right)^{2}\right]^{2}\right\} \\
& \times \frac{v_{e}}{v_{i}}\left(\frac{L}{t v_{e}}\right)^{3} \frac{v_{e} d t}{L}
\end{aligned}
$$

The idealized Eq. (5) is only defined on the interval $t \in$ $\left[L /\left(v_{e}+v_{i}\right) ; L /\left(v_{e}-v_{i}\right)\right]$ and zero otherwise. As mentioned, Eq. (5) relies on the assumptions of complete and instantaneous parallelization and on the laser being horizontally polarized. Equation (5) displays explicitly how the time-of-flight distributions of photoelectrons map both the electron energy by the temporal range covered and the emission anisotropy by the shape of the distribution. For instance, an isotropic distribution $(\beta=0)$ will appear as a decreasing $t^{-3}$ function between two sharp edges, a distribution with $\beta=2$ will display a minimum between the two edges, while a distribution corresponding to $\beta=-1$ will be zero at the edges and display a maximum. The total temporal width where the distribution is nonzero is given by

$$
w=\frac{2 L \times \min \left(v_{i}, v_{e}\right)}{\left|v_{e}^{2}-v_{i}^{2}\right|},
$$

which demonstrates how the Doppler shift imposed by the moving ion beam can be advantageously used to widen the TOF distribution and thereby improve the experimental resolution of the angular anisotropy.

While the analytical expression in Eq. (5) holds the essential features of the observable TOF distribution, the idealized assumption of instantaneous and complete parallelization of the momenta for all electrons is evidently oversimplifying. As an improved approximation, the parallelization may be considered adiabatic [26], meaning that the angular momenta of the electrons are considered conserved during the (still instantaneous) magnetic bending; in this case, the transfer of transverse to longitudinal momentum is governed only by the ratio of the magnetic field strength at the interaction point and in the drift tube.

In the actual experiment, however, also this adiabatic approximation cannot be considered fulfilled, and moreover, the time spent in the inhomogeneous part of the magnetic field cannot be neglected. Additionally, the conditions of the actual experimental situation, such as the finite beam sizes, the temporal laser pulse width, and the presence of small electrical contact potentials on the spectrometer electrodes will affect the observed TOF distribution. Finally, a small fraction of electrons emitted upward may not be transmitted effectively to MCP 1 by the magnetic field. Thus, to obtain a realistic representation of the observed photoelectron distribution, we introduce a parametric Monte Carlo model $P_{\mathrm{MC}}\left(t, \varepsilon_{e}, \beta, \mathbf{p}\right)$ where $\mathbf{p}=\left(A_{0}, A_{1}, L_{d}, \delta t\right)$ holds a set of parameters that accounts for deviations from the idealized distribution, Eq. (5).

In this model, the parallelization of the electron velocities is described by two parameters, $A_{0}$ and $A_{1}$, such that the final transverse velocity of an emitted photoelectron is written as

$$
v_{\mathrm{ef}}^{\perp}=v_{\mathrm{ei}}^{\perp} \sqrt{A_{0}+A_{1} \frac{v_{\mathrm{ei}}^{\perp}}{v_{i}}},
$$

where $A_{0}$ accounts for an adiabatic parallelization and $A_{1}$ for first-order deviations from adiabaticity; the normalization of $v_{\text {ei }}^{\perp}$ to the ion velocity $v_{i}$ is performed to express $A_{1}$ as a unitless number. To describe parametrically the combined effects of the noninstantaneous bending process, the finite beam sizes, the finite laser temporal width, and contact potentials, we further introduce an effective distance $L_{d}$ from the interaction region to the detector and an effective temporal resolution $\delta t$.

For a given parameter set, the Monte Carlo distribution $P_{\mathrm{MC}}\left(t, \varepsilon_{e}, \beta, \mathbf{p}\right)$ is obtained numerically as follows: (a) a set of random inclination angles $\tilde{\theta}$ is generated obeying the general distribution in Eq. (2), together with a set of random azimuth angles $\tilde{\phi} \in[0 ; 2 \pi]$, each set consisting of $N=5 \times 10^{5}$ numbers; (b) the initial electron velocities $\left(v_{\mathrm{ei}}^{\|}\right.$, $v_{\text {ei }}^{\perp}$ ) are calculated according Eq. (3); (c) the final electron velocities after the magnetic bending $\left(v_{\text {ef }}^{\|}, v_{\text {ef }}^{\perp}\right)$ are determined by applying Eq. (7) and energy conservation; (d) the times of flight to the detector are calculated as $t_{e}=L_{d} / v_{\mathrm{ef}}^{\|}$; (e) the resulting set of times $t_{e}$ is histogrammed to form a distribution $P_{\mathrm{MC}}^{0}\left(t_{e}\right)$; (f) the function $P_{\mathrm{MC}}^{0}\left(t_{e}\right)$ is folded with a Gaussian of width (rms) $\delta t$ to obtain the final distribution $P_{\mathrm{MC}}\left(t, \varepsilon_{e}, \beta, \mathbf{p}\right)$. 


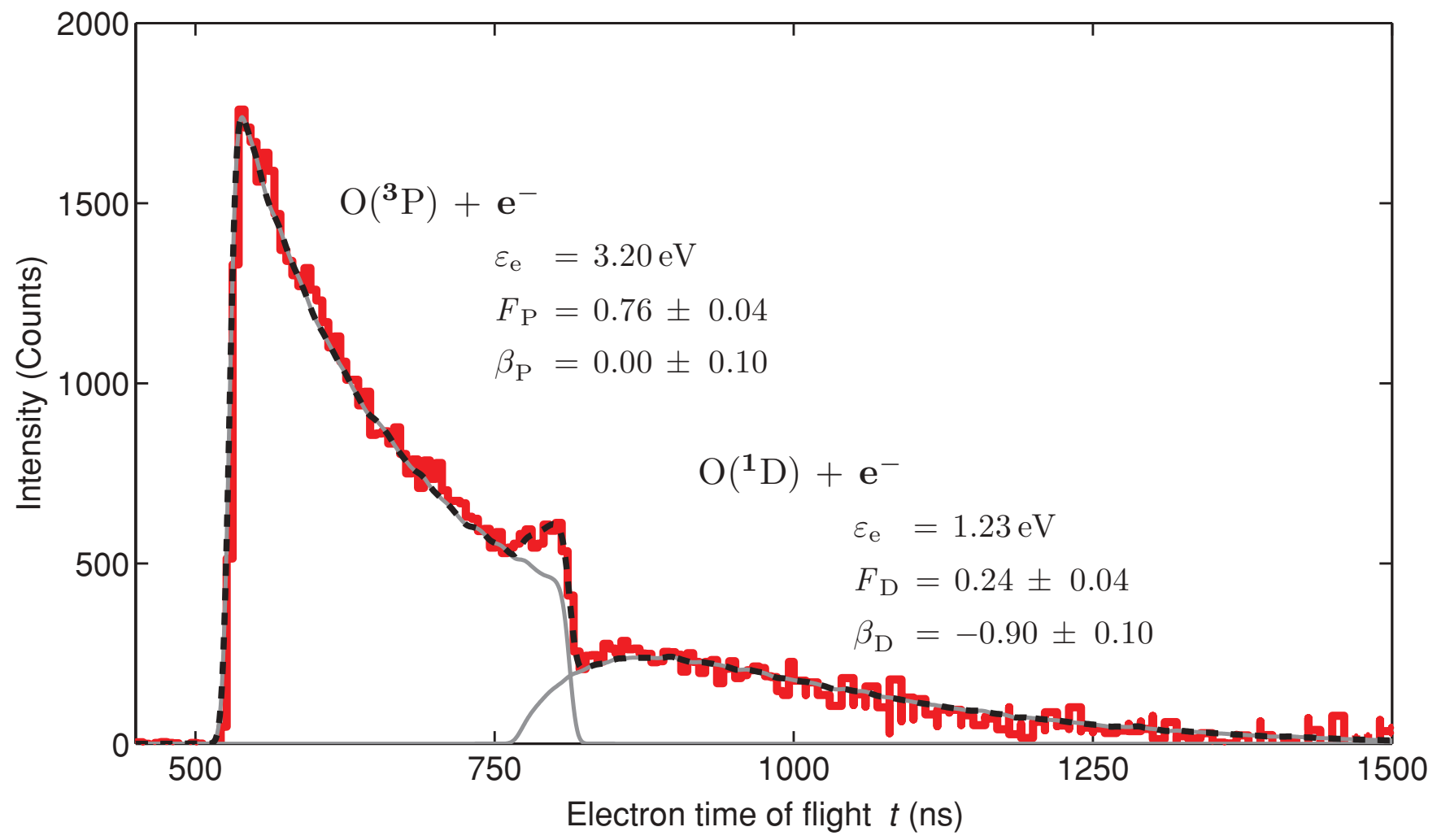

FIG. 3. (Color online) Measured electron time-of-flight distribution after photodetachment of $\mathrm{O}^{-}$at $266 \mathrm{~nm}$. The red (thick) curve shows the experimental distribution, while the dashed black curve shows a fit to the distribution with a Monte Carlo model (see Sec. II B). The gray (thin) curves show the individual contributions from transitions to $\mathrm{O}\left({ }^{3} P\right)$ and $\mathrm{O}\left({ }^{1} D\right)$ to the total fit.

\section{RESULTS AND DISCUSSION}

Figure 3 shows the observed distribution of electron times of flight after photodetachment of $\mathrm{O}^{-}$at $266 \mathrm{~nm}$. The distribution has two distinct components corresponding to transitions into the ${ }^{3} P\left(\varepsilon_{e}=3.20 \mathrm{eV}, t=520-820 \mathrm{~ns}\right)$ and the ${ }^{1} D\left(\varepsilon_{e}=\right.$ $1.23 \mathrm{eV}, t=750-1500 \mathrm{~ns})$ state of atomic oxygen. The TOF distribution for the $\mathrm{O}\left({ }^{3} P\right)$ channel is characterized by rather sharp edges both at high and low times (the fine structure of the ${ }^{3} P$ is not resolved in this experiment) with a smooth decline between them corresponding to a near isotropic $(\beta \sim$ 0 ) electron emission. In contrast, the distribution for the $\mathrm{O}\left({ }^{1} D\right)$ channel shows smooth edges at low and high times with a maximum at $\sim 865 \mathrm{~ns}$ characteristic of a distribution with $\beta \leqslant$ 0 [see Eq. (5)].

The observed TOF distribution can be quantitatively modeled by a sum of two independent components

$$
\begin{aligned}
P_{\exp }(t)= & F_{P} P_{\mathrm{MC}}\left(t, 3.20 \mathrm{eV}, \beta_{P}, \mathbf{p}\right) \\
& +F_{D} P_{\mathrm{MC}}\left(t, 1.23 \mathrm{eV}, \beta_{D}, \mathbf{p}\right),
\end{aligned}
$$

where the evaluation of the Monte Carlo function is described above (Sec. II B). We obtain both the channel fractions $\left(F_{P}\right.$, $\left.F_{D}\right)$ and the anisotropy parameters $\left(\beta_{P}, \beta_{D}\right)$ characterizing the photodetachment dynamics, as well as the parameter set $\mathbf{p}=\left(A_{0}, A_{1}, L_{d}, \delta t\right)$ from a least squares fit of Eq. (8) to the experimental distribution. The dashed black line in Fig. 3 shows the resulting model distribution.
For the transition to the $\mathrm{O}\left({ }^{3} P\right)$ state we obtain

$$
\begin{aligned}
& F_{P}=0.76 \pm 0.04, \\
& \beta_{P}=0.00 \pm 0.10,
\end{aligned}
$$

while for the transition to the $\mathrm{O}\left({ }^{1} D\right)$ state the result is

$$
\begin{gathered}
F_{D}=0.24 \pm 0.04, \\
\beta_{D}=-0.90 \pm 0.10,
\end{gathered}
$$

together with fitted values of the experimental parameters of $A_{0}=(3.8 \pm 0.5) \times 10^{-2}, A_{1}=(4.0 \pm 0.5) \times 10^{-4}, L_{d}=$ $666 \pm 2 \mathrm{~mm}$, and $\delta t=4.7 \pm 0.5 \mathrm{~ns}$.

From the present experiment we thus determine the branching ratio between excited- and ground-state oxygen production at $266 \mathrm{~nm}$ to be $F_{D} / F_{P}=0.32 \pm 0.06$, representing a direct experimental value for this ratio; a rough estimate has previously been given based on the total photodetachment cross section [4]. At lower energy, branching ratios between the fine structure components of the $\mathrm{O}\left({ }^{2} P_{J}\right) \rightarrow \mathrm{O}\left({ }^{3} P_{J}\right)$ transitions have been studied $[6,7,9]$. On the theoretical side, some studies around $266 \mathrm{~nm}$ have only reported the total photodetachment cross section [15-19], while partial channel-resolved cross sections have indeed also been presented in several calculations [20-23] allowing a direct comparison to the present experiment. Thus, the ratio ${ }^{1} D:{ }^{3} P$ after detachment has so far been calculated to $\sim 0.8$ [20] (from their Fig. 3), 0.6 [21] (from their Fig. 4), 0.5 [22] (from their Fig. 4), and most recently $\sim 0.25$ [23] (from their Fig. 3). 
In comparison, the most recent calculation [23] slightly underestimates the experimental result, while the earlier calculations all significantly overestimated the ${ }^{1} D:{ }^{3} P$ ratio.

The present experimental investigation is also the first to address the electron emission anisotropy in the region where both ${ }^{1} D$ and ${ }^{3} P$ states of oxygen are accessible, while several experiments have been performed at lower energy [5-8] (see also Fig. 1). For the transition to ${ }^{3} P$, the angular distribution mimics a pure $s$ wave with $\beta$ close to zero as given in Eq. (9), while the transition to the ${ }^{1} D$ state is accompanied by a negative value of $\beta \sim-0.9$ far away from either pure $s$-wave $(\beta=0)$ or pure $d$-wave $(\beta=1)$ behavior, thus giving clear evidence of superposition of the outgoing waves. Only few calculations $[18,25]$ have given explicit values for $\beta$, and only below $4 \mathrm{eV}$, thus a direct comparison to theory is not possible at this stage. For the transition to the ${ }^{3} P$ state, a simple linear extrapolation to higher energies seems to favor the Cooper-Zare formula [25] over the theory of Stehman and Woo [18], thus giving a value for $\beta_{P}$ close to the experimental value $(0.00 \pm 0.10)$.

$\mathrm{Wu}$ et al. [23] report cross sections resolved both with respect to the excitation of the oxygen atom and the angular momentum of the emerging electron, in particular (from their Fig. 3) at $4.67 \mathrm{eV}: \sigma\left({ }^{3} P+\varepsilon_{e} d\right) \sim 2.2 \mathrm{Mb}, \sigma\left({ }^{3} P+\varepsilon_{e} s\right)$ $\sim 7.2 \mathrm{Mb}$ and $\sigma\left({ }^{1} D+\varepsilon_{e} d\right) \sim 0.7 \mathrm{Mb}, \sigma\left({ }^{1} D+\varepsilon_{e} s\right) \sim 1.8 \mathrm{Mb}$. However, the phases of the outgoing partial waves are not given explicitly, making it presently difficult to judge the effect of interference of the various outgoing waves (see Fig. 1). Thus, it appears as an interesting problem to disentangle the photoelectron anisotropy at elevated photon energies where several different electron waves can interfere.

It should be emphasized that the present experiment is carried out in a static magnetic field of $\sim 50 \mathrm{mT}$, which could possibly affect both the photoabsorption and the subsequent electron-oxygen scattering process. On the other hand, the energy shift in this field is small $\left(\mu_{B} B \sim 3 \mu \mathrm{eV}\right)$ compared to the electron ejection energy $\varepsilon_{e}$, and the electron cyclotron radius is very large $\left(\sqrt{2 m_{e} \varepsilon_{e}} / e B \sim 100 \mu \mathrm{m}\right)$ compared to atomic dimensions. We therefore expect the effect of this static field to be of minor importance.

The present experiment represents a first example where the Doppler shift of electrons emitted from a fast-moving ion beam has been used in combination with the magnetic-bottle technique to study angular distributions of photoelectrons. The possibility of angular resolved measurements using a magnetic-bottle spectrometer was discussed already by Kruit et al. [26] using retarding potentials; however, the Doppler effect has been mostly considered to be an artifact limiting the achievable energy resolution [31]. Photoelectron angular distributions have been studied previously by methods based on rotation of the laser polarization and with a detector at a certain angle $[5,6,8,34]$, and more recently with advanced methods based on photoelectron imaging in two $[35,36]$ and three $[37,38]$ dimensions. The technique described here appears as a simple alternative to the imaging techniques, especially for studies of photodetachment dynamics in systems where the energetics are well known; however, determination of photoelectron energies is of course still feasible using the magnetic-bottle method.

Additionally, as indicated in Fig. 2, the method can be combined with coincident detection of neutral fragments emerging from the photodetachment reaction (possibly with momentum imaging). This would in particular be interesting for studies of molecular systems. Following this experiment, we intend to employ this technique also at higher photon energies as they can be obtained from FLASH.

\section{CONCLUSION}

Summarizing, we have experimentally investigated the photodetachment dynamics of the atomic oxygen anion $\mathrm{O}^{-}$at $266 \mathrm{~nm}(4.67 \mathrm{eV})$ where detachment into $\mathrm{O}\left({ }^{3} P\right)$ and $\mathrm{O}\left({ }^{1} D\right)$ competes. We find a final-state branching of ${ }^{1} D:{ }^{3} P=0.32 \pm 0.06$ and anisotropy parameters of $\beta_{P}=$ $0.00 \pm 0.10$ and $\beta_{D}=-0.90 \pm 0.10$. A recent theoretical calculation [23] predicts a branching ratio of $\sim 0.25$, thus only slightly smaller than the experimental result. For the angular distribution of photoelectrons, a direct comparison with theory is not yet possible. The present experiment invites a deeper theoretical exploration of high-energy photodetachment of oxygen.

The present experiment relies on the use of a fast-moving ion beam which makes it possible to take advantage of the Doppler shift imposed by the moving ion beam on the energies of the emerging photoelectrons to effectively resolve the angular distributions with a magnetic-bottle photoelectron spectrometer. The directed forward motion of the ion beam can also allow universal access to the neutral fragments emerging from the reaction.

\section{ACKNOWLEDGMENTS}

This work has been supported by the Max-Planck Advanced Study Group at CFEL Hamburg and the Max-Planck Society. H.B.P. acknowledges support from the Lundbeck foundation. The effort of R. Treusch, N. Guerassimova, and the technical staff in the FLASH experimental hall is also gratefully acknowledged.
[1] R. P. Wayne, Chemistry of Atmospheres, 3rd ed. (Oxford University, New York, 2000).

[2] D. T. Vier and J. E. Mayer, J. Chem. Phys. 12, 28 (1944).

[3] L. M. Branscomb, D. S. Durch, S. J. Smith, and S. Geltman, Phys. Rev. 111, 504 (1958).

[4] L. M. Branscomb, S. J. Smith, and G. Tisone, J. Chem. Phys. 43, 2906 (1965).
[5] J. L. Hall and M. W. Siegel, J. Chem. Phys. 48, 943 (1968).

[6] F. Breyer, P. Frey, and H. Hotop, Z. Phys. A 286, 133 (1978).

[7] S. J. Cavanagh, S. T. Gibson, M. N. Gale, C. J. Dedman, E. H. Roberts, and B. R. Lewis, Phys. Rev. A 76, 052708 (2007). 
[8] D. Hanstorp, C. Bengtsson, and D. J. Larson, Phys. Rev. A 40, 670 (1989).

[9] K. M. Ervin, I. Anusiewich, P. Skurski, J. Simons, and W. C. Lineberger, J. Phys. Chem. A 107, 8521 (2003).

[10] L. C. Lee and G. P. Smith, J. Chem. Phys. 70, 1727 (1979).

[11] D. M. Neumark, K. R. Lykke, T. Andersen, and W. C. Lineberger, Phys. Rev. A 32, 1890 (1985).

[12] T. Suzuki and T. Kasuya, Phys. Rev. A 36, 2129 (1987).

[13] C. Blondel, C. Delsart, C. Valli, S. Yiou, M. R. Godefroid, and S. Van Eck, Phys. Rev. A 64, 052504 (2001).

[14] P. Hlavenka, R. Otto, S. Trippel, J. Mikosch, M. Weidemüller, and R. Wester, J. Chem. Phys. 130, 061105 (2009).

[15] E. J. Robinson and S. Geltman, Phys. Rev. 153, 4 (1967).

[16] R. J. W. Henry, Phys. Rev. 162, 56 (1967).

[17] K. L. Vo, N. Feaytrier, M. Le Dourneuf, and H. van Regenmorter, J. Phys. B 5, 1506 (1972).

[18] R. M. Stehman and S. B. Woo, Phys. Rev. A 20, 281 (1979).

[19] O. Zatsarinny and K. Bartschat, Phys. Rev. A 73, 022714 (2006).

[20] J. W. Cooper and J. B. Martin, Phys. Rev. 126, 1482 (1962).

[21] W. R. Garrett and H. T. Jackson, Phys. Rev. 153, 28 (1967).

[22] R. L. Chase and H. P. Kelly, Phys. Rev. A 6, 2150 (1972).

[23] J.-H. Wu, J.-M. Yuan, and K. L. Vo, Chin. Phys. 12, 1390 (2003).
[24] G. Miecznik and C. H. Greene, Phys. Rev. A 53, 3247 (1996).

[25] J. Cooper and R. N. Zare, J. Chem. Phys. 48, 942 (1968).

[26] P. Kruit and F. H. Read, J. Phys. E 16, 313 (1983).

[27] A. M. Rijs, E. H. G. Backus, C. A. De Lange, N. P. C. Westwood, M. H. M. Janssen, J. Electron. Spectrosc. Related Phenom. 112, 151 (2000).

[28] M. Förstel et al. (unpublished).

[29] H. B. Pedersen et al., Phys. Rev. A 80, 012707 (2009).

[30] K. Tiedtke et al., New J. Phys. 11, 023029 (2009).

[31] O. Cheshnovsky, S. H. Yang, C. L. Pettiette, M. J. Craycraft, and R. E. Smalley, Rev. Sci. Instrum. 58, 2131 (1987).

[32] S. C. Page, L. Mei, D. Palfreyman, and F. H. Read, Rev. Sci. Instrum. 64, 2574 (1993).

[33] R. Giniger, T. Hippler, S. Ronen, and O. Cheshnovsky, Rev. Sci. Instrum. 72, 2543 (2001).

[34] D. J. Pegg, J. S. Thompson, R. N. Compton, and G. D. Alton, Nucl. Instrum. Methods B40/41, 221 (1989).

[35] A. T. J. B. Eppink and D. H. Parker, Rev. Sci. Instrum. 68, 3477 (1997).

[36] H. Helm, N. Bjerre, M. J. Dyer, D. L. Huestis, and M. Saeed, Phys. Rev. Lett. 70, 3221 (1993).

[37] K. A. Hanold, A. K. Luong, T. G. Clements, and R. E. Continetti, Rev. Sci. Instrum. 70, 2268 (1999).

[38] H. B. Pedersen, M. J. Jensen, C. P. Safvan, X. Urbain, and L. H. Andersen, Rev. Sci. Instrum. 70, 3289 (1999). 